\title{
Some fundamental aspects of elementary gas-surface interactions*
}

\author{
M. Cacciatore $\dagger$ \\ CNR-Centro di Studio per la Chimica dei Plasmi, Dipartimento di Chimica, \\ Universita di Bari, v. Orabona 4, 70126 Bari, Italy
}

\begin{abstract}
Some of the most recent results obtained in theoretical studies on the dynamics of elementary gas-surface processes are presented and discussed. In particular, we give examples of the importance of electronic structure calculations, classical and semiclassical dynamics simulations for understanding the fundamental features of surface reactions relating to plasma processing and plasma-wall interactions.
\end{abstract}

\section{INTRODUCTION}

The interaction of atoms and molecules with surfaces can lead to a great variety of physical and chemical processes of central importance in many research area such as classical catalysis, aerothermodynamics, interstellar chemistry, technology of nuclear reactors. Obviously, heterogeneous processes play a key role in plasma processing and plasma-wall interactions: we mention, among others, the chemisorption of atoms and molecules, surface diffusion, sputtering and ion implantation, desorption of chemisorbed atoms and molecular radicals leading to etching, trapping of molecular fragments on surfaces responsible for film growing. These and other plasma-surface processes are among the most complex processes occurring in plasma reactors. The reaction kinetics of such large chemical systems is generally very complex, but nevertheless the rate determining step is, very often, the dissociative chemisorption of simple molecules, $\mathrm{H}_{2}, \mathrm{CO}, \mathrm{N}_{2}, \mathrm{Cl}_{2}, \mathrm{~F}_{2}, \mathrm{NO}$ and others, on metals and semiconductors. The kinetics of these elementary processes is generally well described, but, in order to reveal the factors that control and eventually enhance the reaction rates, a dynamical description in terms of fundamental steps is necessary.

In the last few years the understanding of the gas-surface dynamics has advanced decisively with the aid of detailed scattering experiments [1] where the use of supersonic molecular beams gives direct access to dynamical effects of the reactions taking place at the surface. In addition, the development of nanosecond, or picosecond, STM techniques [2] has made it possible the real time observation of surface processes. Complementary to the scattering information is the large mass of spectroscopic data (HREELS, IRRAS etc.) now available on the static properties of chemisorbed molecules [3]: equilibrium geometry, bond energies, inter- and intramolecular vibrational frequencies for different specific adsorption sites.

Despite the large amount of information now emerged from such different experimental observations, collisional data, i.e. sticking coefficients, rate constants etc., are very sparse if not absent so that, very often, rate coefficients for heterogeneous processes are taken from similar reactions in the gas-phase. Moreover, a complete understanding of the elementary surface processes is still far from being achieved and a number of fundamental questions are under current investigations. Among the others, the probably most critical aspects concern, on the one hand, the internal state selectivity of the sticking probabilities and, on the other hand the energy transfer mechanisms that control the surface processes. From a practical

*Lecture presented at the 14th International Symposium on Plasma Chemistry, Prague, Czech Republic, 2-6 August 1999, pp. 1809-1918.

$\dagger$ Correspondence: E-mail: cacciatore@area.ba.cnr.it 
point of view, the former aspect is very relevant since the possible variation of the reaction probabilities with the roto-vibrational (and eventually electronic) energy of the active molecule indicates a way to make the process more efficient and selective.

Great progresses in improving the existing collisional data have been made on the theoretical side $[4,5]$. In fact, in the last few years a variety of dynamical methods have been developed to simulate surface processes, ranging from fully ab initio quantum calculations to quasiclassical and semiclassical methods, including Monte Carlo and statistical simulations. Molecular dynamics (MD) simulations have some important features in that molecular dynamics studies predict quantities that can not be easily measured in experimental observations, such as:

- state-to-state surface coefficients and their dependence upon the internal energy content of reactive molecule;

- translational and internal energy distributions in the product states;

- energy exchanged between the surface and the chemical system; and

- reaction mechanism and reaction pathways.

In this paper, these and other features of surface processes relevant to plasma-surface interactions recently explored in MD studies are briefly presented and discussed.

\section{POTENTIAL ENERGY SURFACES FOR MOLECULE-SURFACE INTERACTIONS}

The molecular dynamics simulations imply the solution of two fundamental problems: the first concerns the determination of the forces exerted between the gas-phase particles and the surface atoms, the second problem concerns the development of a suitable collisional model to follow the nuclear motion of the chemical particles interacting with the surface.

The determination of the potential energy surface (PES) where the reaction occurs is a prerequisite for any molecular dynamics calculations and this is addressed, in principle, by using computational methods developed in quantum chemistry for electronic structure calculations [6]. It turns out that the accurate determination of the PES is very difficult, such that the 'true' interaction potential remains rather unknown for even the most elementary heterogeneous systems. The PES is, generally, a multidimensional function which depends, for diatom-surface interaction, upon at least six coordinates: that is the potential surface is too much complex and the number of points to characterise the complete interaction too large for $a b$ initio calculations, either cluster calculations or slab calculations, involving a large number of atoms and electrons. Most electronic structure calculations have been focused on the dissociative chemisorption of $\mathrm{H}_{2}$ and few other diatomic on metals, $\mathrm{Cu}, \mathrm{W}, \mathrm{Ni}, \mathrm{Pd}$ among others [7]. For these elementary systems accurate high-dimensional adiabatic PES are now available at a given interaction surface site as a function of the molecule-surface distance, the intramolecular bond distance and the orientational angles. These quantum calculations have been very useful for understanding the qualitative and quantitative aspects of the dissociative dynamics concerning, in particular, the determination of the 'nature' and height of the reaction barriers, minimum energy path from the molecular to dissociative state, structure of the transition state.

Very recently, electronic structure calculations and PES determinations have been extended on small polyatomics and larger clusters including $\mathrm{Si}$, diamond, metal oxides. These calculations are of great interest for a basic understanding of surface processes of interest in plasma etching, thin film deposition, chemical sensing of solid state microsensors [8].

To give an example of the importance of PES determination in these research fields, here we mention the generalised valence bond (GVB), configuration interaction cluster calculations [9] recently performed to calculate the energy barrier and reaction path for the $\mathrm{C}_{5} \rightarrow \mathrm{C}_{6}$ reaction, which is the last step in the reaction mechanism assumed for growing diamond (100) surface $[10,11]$. The results of this work show that the energy barrier to reaction calculated including the effects of the surface constrains is as small as $\mathrm{E}_{\mathrm{act}}=8.8 \mathrm{kcal} / \mathrm{mol}$. Making use of the vibrational frequencies calculated along the reaction path, the rate constant for the reaction was also estimated in the transition state approximation. It turns out that the TST rate is several orders of magnitude larger than any other intermediate reactions in the growth mechanism. 
Since accurate $a b$ initio PES are available for a very limited number of heterogeneous systems, very often one has to develop approximate semiempirical interaction potentials using all the experimental and $a b$ initio data available in the literature (see, for example [24]). In this context, the CO/Pt system offer a very interesting example. This system is, in fact, among the most extensively studied system: virtually all the experimental techniques have been applied to probe the chemisorbed molecular states, including LEED, TDS, EELS, IR and, most recently, He scattering experiments [12]. All this mass of spectroscopic data can be used to build up a realistic PES for CO/Pt interaction. Assuming a trial interaction potential as a sum of pair-wise interactions between the gas-phase atom $i(\mathrm{C}, \mathrm{O})$ and the Pt surface atom $\alpha$ :

$\mathrm{V}_{\mathrm{CO} / \mathrm{Pt}}(\mathrm{R}, \mathrm{r})=$

$\sum_{i=1}^{2} \sum_{\alpha=1}^{N} \exp \left(-\beta_{i} \cdot \mathrm{R}_{i \alpha}\right)\left(\mathrm{D}_{i} \exp \left(-\beta_{i} \mathrm{R}_{i \alpha}\right)-\mathrm{E}_{i}+\mathrm{F}_{i} \cdot \mathrm{R}_{i \alpha}+\mathrm{G}_{i} \cdot \mathrm{R}_{i \alpha}^{2}\right)+\mathrm{D}_{\mathrm{CO}}\left(1-\exp \left(-\beta\left(\mathrm{r}-\mathrm{r}_{\mathrm{eq}}\right)\right)\right)^{2}$

the free parameters can be determined so as to reproduce all the observed spectroscopic properties of CO chemisorbed on Pt. This can be achieved performing a normal mode analysis [13] by numerical diagonalization of the second derivatives dynamical matrix and varying the potential parameters until a good convergence is reached between the experimental and the calculated frequencies. The total reactive PES is then obtained by adding to the $\mathrm{V}_{\mathrm{CO} / \mathrm{Pt}}$ potential the potential for the $\mathrm{C} / \mathrm{Pt}$ and $\mathrm{O} / \mathrm{Pt}$ interactions. Each pair potential must be switched appropriately from the molecular to the atomic interactions.

Figure 1 shows the contour map of the total optimised PES for CO interacting perpendicular in the bridge site of $\operatorname{Pt}(111)$.

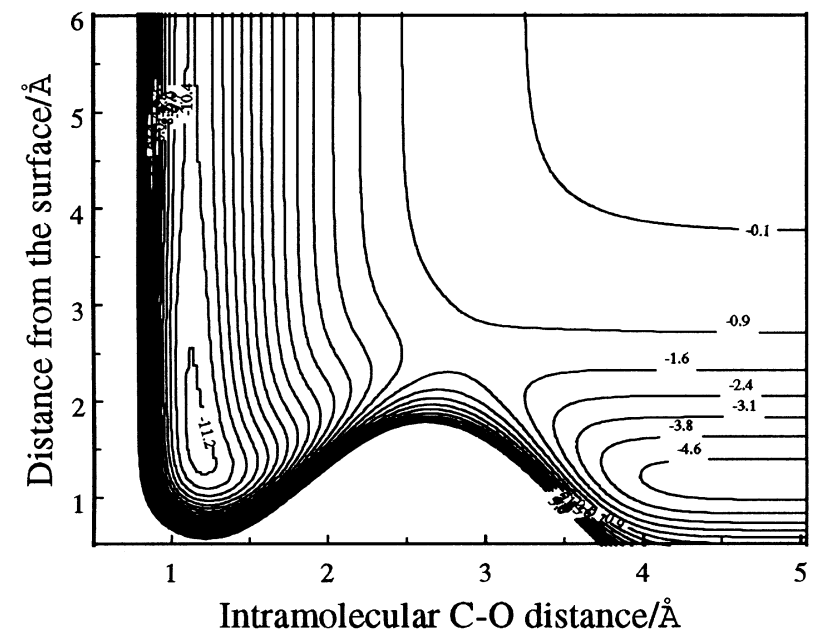

Fig. 1 Contour map for CO interacting perpendicular on the bridge site of $\operatorname{Pt}(111)$.

The PES clearly shows the existence of a chemisorption well for CO located at a distance of $1.5 \AA$ from the surface and with a well depth of about $1.1 \mathrm{eV}$. Both these values are in very good agreement with the experimental ones. According to this PES, the transition state is far into the exit channel with the $\mathrm{C}-\mathrm{O}$ bond in a largely stretched configuration. As a consequence of the PES topology we would expect a strong influence of the vibrational energy content of $\mathrm{CO}$ in the dissociation process. This new PES, has been used to simulate $\mathrm{CO}$ dissociation and $\mathrm{CO}$ formation via the Eley-Rideal (E-R) mechanism on a Pt(111) surface [13].

In plasma processing simulation a great impact have had the semiempirical potential developed for the first time by Stillinger and Weber (SW) for the pair interactions between $\mathrm{Si}-\mathrm{Si}, \mathrm{Si}-\mathrm{F}$ and F-F [14]. This potential was formulated so as to correctly describe the bulk properties of the silicon surface and the spectroscopic properties of the gas-phase $\mathrm{F}_{2}$ and $\mathrm{SiF}_{x}$ species and it has be used in several works to simulate the atomic and molecular fluorine etching of $\mathrm{Si}(100)-2 \times 1$ reconstructed surface $[15,16]$. The behaviours of the atomic fluorine etching rate predicted in these works have not been confirmed experimentally in that the predicted rate exhibits a strong dependence with the kinetic energy of the 
etching species and a maximum for the clean surface. Recently, the SW potential has been implemented by fitting the parameters for the heterogeneous $\mathrm{Si}-\mathrm{F}$ interactions to the energies obtained in ab initio GVB cluster calculations $[17,18]$. A semiempirical potential has been developed also for $\mathrm{Si}-\mathrm{H}$ interactions and it has been used in MD simulations to investigate the reaction of $\mathrm{SiH}$ and $\mathrm{SiH}_{3}$ radicals with hydrogenated $\operatorname{Si}(001)$ surfaces [19].

\section{MOLECULAR DYNAMIC SIMULATIONS}

The main object of molecular dynamics approach to surface processes is to calculate the state-selected sticking probability $\mathrm{S}_{0}\left(\mathrm{v}, \mathrm{j} ; \theta, \varphi ; \mathrm{E}_{\mathrm{kin}}, \mathrm{T}_{\mathrm{S}}\right)$ for a specific elementary surface process as a function of variables relevant to the initial conditions of the reagents: the incident kinetic energy, $\mathrm{E}_{\text {kin }}$, the initial orientation angles, $(\theta, \varphi)$, the initial roto-vibrational $(\mathrm{j}, \mathrm{v})$ state of the molecule hitting the surface and the surface temperature, $\mathrm{T}_{\mathrm{S}}$. Final aim is then to study the influence of the structural and chemical properties of the surface and of the molecule on the sticking coefficient $S_{0}$. Such detailed quantity can not be easily measured in experiments, due to the persisting difficulty in controlling the internal states of the molecules in the incident beam as well as in detecting the energy distribution in the final states of the products formed at the surface and reflected into the gas-phase. Such a difficulty has been the cause of ambiguity in assigning the activation energies for chemisorption processes where the vibrational motion of the dissociating molecule is coupled with the reaction coordinate. Paradigmatic is, in this respect, the intense discussion developed over the last decades on the assignment of the activation energy for the dissociative chemisorption of $\mathrm{H}_{2}(\mathrm{v}, \mathrm{j})$ on $\mathrm{Cu}$ surfaces [7].

From the calculated state-to-state coefficient $\mathrm{S}_{0}$ different averaged quantities can be obtained. Thus, the state-averaged sticking coefficient $\left\langle\mathrm{S}_{0}\left(\theta, \varphi ; \mathrm{E}_{\text {kin }}, \mathrm{T}_{\mathrm{S}}\right)>\right.$, usually measured in scattering experiments, is obtained by averaging over a known, not necessarily Boltzmann population distribution $n_{v}$ and $n_{j}$ of the $(\mathrm{v}, \mathrm{j})$ states. The statistical rate constant $\mathrm{K}\left(\mathrm{T}_{\mathrm{S}}\right)=\mathrm{K}_{\text {coll }} \times<\mathrm{S}_{0}\left(\mathrm{~T}_{\mathrm{S}}\right)>$ used in kinetic modelling can be obtained by further averaging $\mathrm{S}_{0}$ over the angles and the Maxwell energy distribution of the reagent at a given translational temperature.

The $a b$ initio calculation of the state-to-state $\mathrm{S}_{0}$ coefficient is not an easy task. The multidimensional nature of the collisional system from one hand, and the large number of degrees of freedom introduced by the presence of the solid increase considerably the complexity of the collision dynamics. In order to describe the most important surface phenomena (desorption and sticking, diffusion, inelastic and reactive scattering) we need to include in the collisional model the molecular properties of the reactive molecule, such as the rotational, vibrational, and, eventually, electronic states, as well as the surface properties: lattice geometry, phonon distribution and electron properties, surface corrugation. From these brief considerations it is evident that the number and the complexity of effects to account for in molecular dynamics is very high.

The most natural way to simulate a surface process would be to solve the electronic structure problem and the dynamics of the nuclear motion of the chemical system simultaneously in a self consistent way. This approach has been followed by Car \& Parrinello [20] who developed for the first time a fully $a b$ initio classical dynamics simulation of surface processes. Subsequently, this method has been implemented [21] making use of the pseudo potential approximation used in density functional theory-local density approximation (DFT-LDA) which has made it possible the simulation of elementary surface reactions to systems involving many hundred of atoms. This method has been applied to a restricted number of heterogeneous systems, including the dissociative chemisorption of chlorine molecules on $\mathrm{Si}(100)-2 \times 1$ surface [22], a basic reaction in the silicon etching via plasma. The results obtained in this study have confirmed, on firm basis, the simple mechanism usually assumed for bond breaking and bond making at surface, according to which dissociative chemisorption occurs because of a charge transfer from the surface electrons, or from other molecular orbital, to the first unoccupied antibonding orbital of the interacting molecule. This is the mechanism for the dissociation reaction of $\mathrm{Cl}_{2}$ on silicon. Indeed, the electronic charge density flows calculated at various interaction times of $\mathrm{Cl}_{2}$ approaching parallel the Si surface show that, at closer distances from the surface, there is a net charge accumulation around the molecule due to the filling of the LUMO 3p$\sigma_{\mathrm{u}}^{*}$ orbital, while a smaller charge depletion of the HOMO $3 \mathrm{p} \pi_{\mathrm{g}}^{*}$ orbital is observed. A further important result obtained in this high impact 
work is that the chlorine molecule with an impact energy of $1 \mathrm{eV}$ dissociates spontaneously in the parallel configuration, while there is a small activation energy for dissociation, $\approx 0.8 \mathrm{eV}$, with the appearing of a short lived precursor state, when $\mathrm{Cl}_{2}$ approaches the $\mathrm{Si}$ surface in the perpendicular configuration. Although the availability of highly parallelised supercomputers, calculations of such high complexity are still extremely costly so that computational scheme of such complexity at moment can not be used for determining surface coefficients requiring the simulation of a large number of collisional events.

On the contrary, collisional data for surface chemical processes can be obtained using collisional models computationally less involved compared to the fully ab initio method, as in quasi-classical and semiclassical models. In particular, the semiclassical approach [23,24] can be very useful in that this method enable us to describe the surface processes at a very detailed level, including the energy exchange mechanisms between the atom/molecules and the substrate generally not considered in fully quantum simulations, and, at the same time, to calculate the sticking coefficients with a reasonable accuracy. The most important feature of the semiclassical approach is the definition and calculation of an effective time-dependent hamiltonian, $\mathrm{H}_{\mathrm{eff}}$, which couples the dynamics of the nuclear motions of the chemical particles, treated in the classical approximation, with the dynamics of the surface electrons and the vibrational motions of the lattice atoms treated in the quantum approximation. Thus:

$\mathrm{H}_{\mathrm{eff}}\left(t, T_{\mathrm{S}}\right)=\mathrm{H}_{\mathrm{eff}}^{\mathrm{ph}}+\mathrm{H}_{\mathrm{eff}}^{\mathrm{e}-\mathrm{h}}=\left\langle\Psi_{\mathrm{ph}}\left|\mathrm{V}_{\mathrm{int}}\right| \Psi_{\mathrm{ph}}\right\rangle+\left\langle\Psi_{\mathrm{el}}\left|\mathrm{V}_{\mathrm{C}}\right| \Psi_{\mathrm{el}}\right\rangle$

where $t$ is the interaction time and $T_{\mathrm{S}}$ the surface temperature, $<\Psi_{\mathrm{ph}} \mid$ and $<\Psi_{\mathrm{el}} l$ are the wavefunctions of the phonon and electronic states of the surface respectively, $\mathrm{V}_{\text {int }}$ is the interaction potential between the lattice atoms and the gas-phase atoms, $\mathrm{V}_{\mathrm{C}}$ is the coulomb potential between the electrons of the surface and the electronic charge transferred from the metal to the molecule. This excess charge can be determined in electronic structure calculations and is responsible of the excitation/de-excitation processes of the metal electrons near to the Fermi level. The total hamiltonian for the nuclear motion of the gas-phase species is obtained by adding to the usual hamiltonian for the free particles the effective hamiltonians arising from the gas-surface interactions.

Despite some complexity in the formulation, the semiclassical model is computationally affordable, yet providing a realistic description of the gas-surface interactions in their full dimensionality.

Semiclassical sticking coefficients have been calculated for the dissociative chemisorption of $\mathrm{H}_{2}, \mathrm{D}_{2}$, $\mathrm{CO}, \mathrm{N}_{2}, \mathrm{O}_{2}$ on different surfaces [24-29]. In these studies several features of the dissociation reaction have been discussed and pointed out. It turns out that these reactions are vibrationally activated, such that the vibrational energy content of the impinging molecule is the most efficient energy source for surmounting the activation barriers to dissociation. This aspect of the dissociative chemisorption is a very fundamental one and it has been discussed for decades before arriving to firm conclusions. Due to obvious experimental difficulties, quite often the $S_{0}(v)$ coefficients can be determined only on a theoretical ground. The calculated semiclassical $\mathrm{S}_{0}(\mathrm{v})$ coefficients for the dissociative chemisorption of $\mathrm{H}_{2}, \mathrm{D}_{2}, \mathrm{~N}_{2}$ exhibit a significant dependence upon the initial vibrational quantum number of the impinging molecule, such that vibrationally excited molecules dissociate faster than molecules in the ground vibrational level. The dependence of $\mathrm{S}_{0}(\mathrm{v})$ upon the vibrational quantum number is rarely considered in kinetic modelling of plasma-surface processes, although the use of the state-to-state coefficients obtained from ab initio calculations could have a great impact on the reactive kinetics of non-equilibrium plasma where the active molecules, before and after the wall interactions, are generally produced in vibrationally pumped distributions.

Surface processes can be assisted either by phonon or electron excitation processes in the substrate. Within the semiclassical method both these effects are included in the dynamics, so that the relative importance of the two mechanisms can be pointed out.

This effect has been explored for the $\mathrm{H}_{2}$ dissociation on a copper surface [24]. In Figure 2 it is reported the energy fraction transferred to the vibrational phonon modes of the atoms in the lattice together with the excitation energy of the electrons above the Fermi level as a function of the interaction time for a typical collisional event $\left(1 \tau=10^{-14} \mathrm{~s}\right)$. From the reported results it can be noticed that in the shorter interaction times the energy transferred to the electrons increases rapidly, while a small phonon perturbation. On the other hands, in the stationary state conditions the total energy accommodation is 


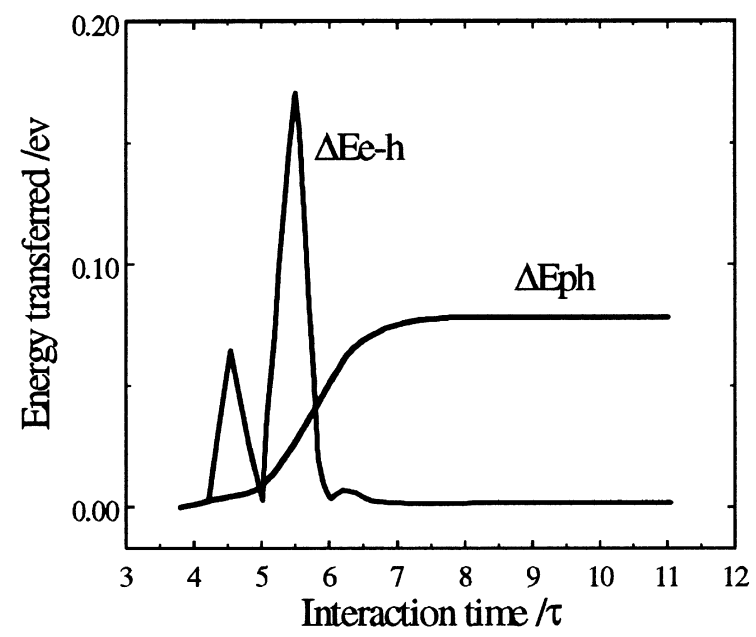

Fig. 2 Energy transfer to the phonons $\Delta \mathrm{Eph}$ and to the electrons $\Delta \mathrm{Ee}-\mathrm{h}$ as a function of the interaction time for $\mathrm{H}_{2}$ in $\mathrm{v}=2, \mathrm{j}=0$ colliding with $\mathrm{Cu}(111)$. The impact energy is Ekin $=1.5 \mathrm{eV}$.

mainly due to the phonon excitation mechanism while the electrons are almost completely deexcited.

The electron-hole pair excitation has, however, an indirect effect on the phonon excitation, in the sense that when the electron excitation mechanism is discarded the energy exchanged with the surface atoms becomes smaller. The concomitant effect of the phonon and of the electron excitation processes has a strong influence on the dissociation probability.

It turns out that, at low impact energies, the sticking coefficient $\mathrm{S}_{0}$ of $\mathrm{H}_{2}$ and $\mathrm{D}_{2}$ on $\mathrm{Cu}$ is larger when both mechanisms are considered in the simulation. In fact, when each of the two energy loss mechanisms is activated the residence time of the molecule on the surface increases so that the probability for the molecule to find the reaction channel to dissociation also increases.

Besides the dissociative chemisorption of diatomic molecules, the atom recombination on metals has attracted considerable attention. In plasma reactors this process can be an effective channel for producing vibrationally excited molecules as well as an effective atom loss mechanism at wall. The large exothermic energy released in this reaction can be partitioned among the surface substrate and the translational and internal states of the formed molecules: depending on the energy partitioning, molecules can be formed in vibrationally, rotationally or translationally excited states. Therefore, the energy exchanged mechanism between the molecule and the substrate play a critical role in the recombination dynamics and, specifically, on the vibrational state-selected recombination coefficient $\gamma(\mathrm{v})$.

Very recently, a detailed semiclassical study on the oxygen atom recombination on silica surfaces via the Eley-Rideal and the Langmuir-Hinshelwood (L-H) mechanism has been performed which have revealed interesting aspects of recombination process [28].

In Figure 3 there are reported the reaction probabilities for the different reaction pathways for the E-R mechanism as a function of the impact energy of the gas-phase oxygen atom. The products for channels $\{\alpha\}-\{\gamma\}$ are, respectively, $\mathrm{O}_{2}(\mathrm{v}, \mathrm{j})$, Ogas + Ogas (inelastic), Oad + Ogas/(Ogas + Oad) (adsorption-desportion), $\mathrm{Oad}+\mathrm{Oad} / \mathrm{O}_{2} \mathrm{ad}$ (trapping). As it can be noticed, the surface is rather inert for molecular oxygen formation, and this is in agreement with the low catalytic activity of silica. Instead, the most active processes are the inelastic channel and the adsorption/desorption processes where one of the two oxygen atoms desorbes in the gas-phase while the other is adsorbed.

The activation of the adsorption processes $\{\gamma\}$ and $\{\delta\}$ at higher energies could have important consequences either for the surface damage, due to the large energy released to the surface, and the surface reactivity due to the changes in the phonon and electronic structure induced by the adsorbed species.

The recombination coefficient $\gamma_{\mathrm{E}-\mathrm{R}}$ obtained from the calculated recombination probabilities is 


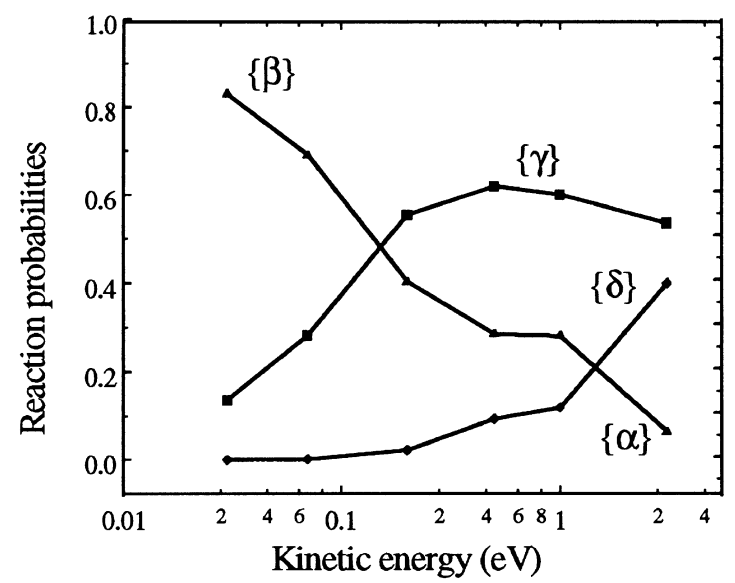

Fig. 3 Reaction probabilities for products $\{\alpha\}-\{\gamma\}$ in the reaction: $\mathrm{O}_{\text {gas }}+\mathrm{O}_{\mathrm{ad}}+$ silica $\rightarrow$ products + silica (see text for details).

$\gamma_{\mathrm{E}-\mathrm{R}}=0.029$ at $T_{\mathrm{S}}=1000 \mathrm{~K}$, in very good agreement with the experimental value reported in literature. On the other hands, the experimental recombination coefficient reported at $T_{\mathrm{S}}=600 \mathrm{~K}$ can be matched assuming that the reaction occurs via the $\mathrm{L}-\mathrm{H}$ mechanism. This result would confirm the switching of the recombination mechanism from $\mathrm{E}-\mathrm{R}$ to $\mathrm{L}-\mathrm{H}$ in the same manner as decreasing the surface temperature. A further interesting result obtained in this work concerns the total energy partitioning in the recombination reaction. It turns out that the largest fraction of the reaction exothermicity is primarily shared among the vibrational and the rotational motions of the formed $\mathrm{O}_{2}$ molecules, while only a small fraction of the total excess energy is converted as heat flux to the surface. As a consequence, $\mathrm{O}_{2}$ is formed in highly vibrationally excited states. From the calculated vibrational energy distribution the probability for $\mathrm{O}_{2}$ formation in a specific vibrational state has also been obtained, as well as the state-to-state recombination coefficients $\gamma(\mathrm{v})$. The influence of the surface coverage and of the adsorption site on the recombination dynamics were also investigated in this work [28].

Surface processes relevant to plasma-etching and plasma CVD have been simulated in many classical MD calculations with the use of semiempirical and ab initio based semiempirical potentials for the $\mathrm{Si}-\mathrm{H}$ and $\mathrm{Si}-\mathrm{F}$ interactions $[17,19,30]$. The sticking coefficient for $\mathrm{F}$ atom abstraction in $\mathrm{F}_{2}-\mathrm{Si}(100)$ interactions has been recently calculated in a wide range of collisional energies [31]. The overall qualitative agreement with the experimental data determined in molecular beam experiments was good. The dependence of the sticking coefficient upon the kinetic energy shows that the reaction is translationally activated, with a negligible effect of the internal energy of the $F_{2}$ molecules. Some interesting results concerning the reaction mechanism and the translational energy distributions of $\mathrm{F}$ and $\mathrm{F}_{2}$ ejected inelastically in the gas-phase are reported and discussed with respect to the issues of implementing the Si etching process.

\section{FINAL REMARKS}

Recent developments of complex collisional models and advances in computing science have made it possible MD simulations of elementary surface reactions relevant to many research areas. In particular, the dissociative chemisorption of diatomic molecules and atom recombination on metals, $\mathrm{Si}$ and metal oxides can now be described at a very detailed atomistic level. For these reactions, state-selected surface coefficients, angular and energy distributions in the product state and reaction pathways can be determined performing quantum mechanical, semiclassical and classical MD simulations at different levels of approximations. Semiclassical calculations have some advantages in that the energy exchange mechanisms between the chemical system and the phonons e/o electrons of the substrate are included in the dynamics, while in quantum mechanical simulations the surface is generally assumed rigid. Instead, classical and semiclassical models have some physical constraints so that simulations within these approximations can be performed for spontaneous reactions, or for activated reactions in the collisional regime above the activation barrier. In the energy range below the barrier, of importance for many 
thermal processes, the reaction occurs only via tunnelling and this requires the quantization of at least the reaction coordinates.

A part from $a b$ initio fully quantum calculations performed for prototype systems, large scale MD simulations are usually carried out utilising semiempirical adiabatic potentials based on experimental data e/o $a b$ initio points obtained from electronic structure calculations. As a matter of fact, the determination of a 'realistic' PES is the most critical problem to solve in MD studies, such that interaction potentials constructed from different data sources may have different behaviours. As a consequence, collisional data for heterogeneous systems are not necessarily convergent, their accuracy being primarily determined from the accuracy of the PES assumed in the dynamics. A further limiting factor for the reliability of these data lies on the 'adiabatic' character of the available PES, so that surface processes involving electronic excited states that could be energetically accessible for some heterogeneous systems can not be explored in the simulations. Though the inclusion of such effects add more complexity and computational time requirements for MD calculations, the impact and importance of approximate, still complex simulations of elementary gas-surface processes is clear and it will become more evident in the next future with the accessibility of fully dimensional $a b$ initio calculations.

\section{ACKNOWLEDGEMENTS}

This work has been supported by the Italian space agency (ASI). The author wish to thank G. D. Billing for fruitful collaboration and discussions.

\section{REFERENCES}

1 H. A. Michelsen, C. T. Rettner, D. J. Auerbach. In Surface Reactions (R. J. Madix, ed.) p. 123. Springer (1993).

2 H. Brune, J. Wintterlin, J. Trost, G. Ertl, J. Wichers, J. Behm. Phys. Rev. 68, 624 (1992); Chem. Phys. 99, 2128 (1993).

3 D.A. King, D. P. Woodruff, eds. The Chemical Physics of Solid Surfaces and Heterogeneous Catalysis, Vol. 2-4. Elsevier (1984).

4 A. E. DePristo, A. Kara. Adv. Chem. Phys. 77, 163 (1990).

5 G. P. Brivio, T. B. Grimley. Surf. Sci. Rep. 17, 1 (1993).

6 (R. M. Lambert, G. Pacchioni, eds) Chemisorption and Reactivity on Supported Clusters and Thin Films NATO Asi Series (1997).

7 A. Groß, S. Wilke, M. Scheffler. Phys. Rev. Lett. 75, 2718 (1995); A. Groß, B. Hammer, M. Scheffler, W. Brenig. Phys. Rev. Lett. 73, 3121 (1994); M. Kay, G. R. Darling, S. Holloway, J. A. White, D. M. Bird. Chem. Phys. Lett. 245, 311 (1995); B. Hammer, M. Scheffler, K. W. Jacobsen, J. K. Norskov. Phys. Rev. Lett. 73, 1400 (1994).

8 F. Boccuzzi, A. Chiorino, S. Tsubota, M. Haruta. Sensors Actuators B24-25, 540 (1995).

9 C. B. Musgrave, S. J. Harris, W. A. Goddard III. Chem. Phys. Lett. 247, 359 (1995).

10 S. J. Harris, D. G. Goodwin. J. Phys. Chem. 97, 23 (1993).

11 C. E. Johnson, W. A. Weimer, F. M. Cerio. J. Mat. Soc. 7, 1427 (1992).

12 A. M. Lahee, J. P. Toennies, Ch. Wöll. Surf. Sci. 177, 371 (1986).

13 E. Christoffersen, M. Cacciatore. Work in preparation.

14 F. H. Stillinger, T. A. Weber. Phys. Rev. B31, 5262 (1985); J. Chem. Phys. 88, 5123 (1988).

15 F. H. Stillinger, T. A. Weber. Phys. Rev. Lett. 62, 2144 (1989); T. A. Weber, F. H. Stillinger. J. Chem. Phys. 92 , 6239 (1990).

16 T. A. Schoolcraft, B. J. Garrison. J. Am. Chem. Soc. 113, 8221 (1991).

17 C. J. Wu, E. A. Carter. Phys. Rev. B45, 9065 (1992).

18 L. E. Carter, E. A. Carter. Surf. Sci. 360, 200 (1996).

19 T. Ohira, O. Ukai, T. Adachi, Y. Takeyuchi, M. Murata. Phys. Rev. B52, 8283 (1995).

20 R. Car, M. Parrinello. Phys. Rev. Lett. 55, 2471 (1985); M. E. Tuckermann, M. Parrinello. J. Chem. Phys. 101, 1302 (1994).

21 M. C. Payne, M. P. Teter, D. C. Allan, T. A. Aries, J. D. Joannopoulos. Rev. Mod. Phys. 64, 1045 (1992). 
22 I. S̆tich, M. C. Payne, A. De Vita, M. J. Gillan, L. J. Clarke. Chem. Phys. Lett. 212, 617 (1993); A. De Vita, I. Štich, M. J. Gillan, M. C. Payne, L. J. Clarke. Phys. Rev. Lett. 71, 1276 (1993).

23 G. D. Billing. Comput. Phys. Rep. 12, 383 (1990).

24 M. Cacciatore, G. D. Billing. Surf. Sci. 232, 35 (1990).

25 G. D. Billing, M. Cacciatore. Faraday Discuss. 96, 33 (1993).

26 M. Cacciatore, G. D. Billing. Pure Appl. Chem. 68, 1075 (1996).

27 G. D. Billing, M. Cacciatore. Chem. Phys. Lett. 113, 23 (1985); Chem. Phys. 103, 137 (1986).

28 M. Cacciatore, M. Rutigliano, G. D. Billing. J. Therm. \& Heat Transf. 13, 195 (1999).

29 F. Y. Hansen, N. E. Henriksen, G. D. Billing. Surf. Sci. 324, 55 (1995).

30 S. Ramalingham, D. Maroudas, E. S. Aydil. J. Appl. Phys. 84, 3895 (1998).

31 L. E. Carter, E. A. Carter. J. Phys. Chem. 100, 873 (1996); L. E. Carter. Surf. Sci. 323, 39 (1995). 